\title{
Deciphering cell's robustness by a multi-scale framework integrating cell cycle and metabolism in yeast
}

\author{
L. van der Zee, T.D.G.A. Mondeel, H. Westerhoff, M. Barberis* \\ Swammerdam Institute for Life Sciences, University of Amsterdam, Amsterdam, The Netherlands \\ *e-mail:M.Barberis@uva.nl
}

Key words: Boolean modeling, constraint-based modeling cell cycle, metabolism, budding yeast

Motivation and Aim: Cell cycle and metabolism are coupled networks. For example, cell growth and division require synthesis of macromolecules which is dependent on metabolic cues. Conversely, metabolites involved in nucleotide and protein synthesis are fluctuating periodically as a function of cell cycle progression. Although computational models of these networks are being developed for some time, to date no effort has been made to integrate these two systems in any organism. We aim to investigate cell cycle robustness by generating the first multi-scale model that integrates cell cycle with metabolism, and investigating their bidirectional regulation. Connections among these two biochemical networks have been recently elucidated in budding yeast. However, high-throughput and manually curated studies point at many more physical interaction, which relevance for precise cell cycle timing remains unknown.

Methods and Algorithms: A framework is presented that integrates a Boolean cell cycle model with a constraint-based model of metabolism, incorporating mechanistic and highthroughput interactions. Directionality and effect are incorporated for the mechanistic interactions. Conversely, as this information is unknown for the high-throughput interactions, an informed optimization algorithm has been developed to generate models that can incorporate it iteratively.

Results: To verify the results of the informed optimization algorithm against metabolomic data, changes in flux through a number of metabolic pathways are compared to metabolic pathway enrichment time-series. The multi-scale model predicts expected changes in a number of pathways, ranging from amino-acid to pentose phosphate to lipid metabolism. Many model variants that differ in number and directionality of interactions robustly predict the effect of definite cell cycle-metabolism pairs. Furthermore, the integrative model shows a temporal export of acetate, pyruvate and alanine, reminiscent of yeast metabolic oscillations.

Conclusion: Altogether, our multi-scale framework is able to integrate computer models of biological networks with high-throughput data, to capture the functional connectivity among their elements that ultimately results in systems robustness.

Acknowledgements: Supported by the SILS Starting Grant of the University of Amsterdam, UvA and by the UvA-Systems Biology Research Priority Area Grant.

\section{References}

1. Barberis M., Todd R.G., van der Zee L. (2017) Advances and challenges in logical modeling of cell cycle regulation: perspective for multi-scale, integrative yeast cell models. FEMS Yeast Research. 17(1). 